7. Yu, H.-G. \& Koshland, D. Cell 123,397-407(2005)

8. Lee, B.H.\&Amon, A.Sdence 300, 482-486 (200B).

9. Kerrebrock A.W, Miyazaki, W. K, Birnby, D. \& Orr-Weaver, T. L Genetics 130, 827-841 (1992).

10. Kitailma, T. S, Kawashima, S.A.\& Watanabe, Y.Nature 427,510-517(2004).
11. Marston, A.L, Tham, W.-H, Shah, H.\& Amon, A.Sdence 303, 1367-1370 (2004).

12. Rabitsch, K P.etal. Curr. Biol. 14, 287-301(2004).

13. Tang, Z.et al. Dev. Cell doit101016/jencel 200603010 (2006)

14. Brar, G. A. et a. Nature dob101038/nature04794 (2006)

\title{
MATERIALS SCIENCE
}

\section{Polymers show they're metal}

\author{
Richard Friend
}

\section{Although certain polymers have long been known to conduct electricity, they seemed to differ from metals in other electronic and optical properties. A new form of polymer turns that relation on its head.}

The fact that the polymer polyacetylene reaches metallic levels of conductivity when chemically doped caused quite a stir when it was first reported ${ }^{1}$ in 1977 . This was not just because conducting polymers might be of great practical use, but also because the underlying physics came as a surprise: the apparently free movement of electrons along the carbon-based backbone of a polymer chain was not widely anticipated. The discovery attracted the attention of many chemists, materials scientists and physicists, and by the mid-1990s several practical conducting polymeric materials had emerged.

Despite their metal-like conductivities, these polymers did not pass muster as metals in other important respects. On page 65 of this issue, Lee et $a l^{2}$ supply missing evidence that supports the prevailing view that it is structural disorder, which is very hard to eliminate in polymeric materials, that prevents making real metals of polymers ${ }^{3,4}$. The authors' doped, conducting form of polyaniline, synthesized to produce a more ordered material than was previously available, shows both the electrical and the optical properties required of a true metal.

Although the electrical conductivity of this form of polyaniline is, at about $10^{3}$ siemens per centimetre at room temperature, not particularly high (the conductivity of copper at the same temperature is, for instance, around $\left.6 \times 10^{5} \mathrm{~S} \mathrm{~cm}^{-1}\right)$, it increases steadily as temperature is lowered, and by 4 kelvin has risen by a factor of about 2.5. Such behaviour is a hallmark of a metal: the conduction electrons that carry the electrical current within a metal travel as free particles, and it is the rate at which these electrons are scattered that limits conductivity. Because the dominant source of scattering is the thermal vibrational motions of the atoms, as temperature falls, scattering is frozen out, and so conductivity increases. At very low temperatures, the conductivity saturates at a level set by scattering due to residual disorder.

Another defining feature of a metal is its optical reflectivity, which gives, for instance, silver and aluminium their familiar shine. Light is reflected from a metallic surface when its frequency lies below the characteristic oscillation frequency (the 'plasma frequency', $\omega_{\mathrm{p}}$ ) of electrons in a metal. (This frequency is given by the expression $\omega_{p}^{2}=n e^{2} / \epsilon m$, where $n$ is the number density of conduction electrons, $m$ the electron mass and $\epsilon$ is the background dielectric constant.) In this case, conduction electrons are able to move fast enough to screen out incident electromagnetic radiation and, mathematically, the real part of the complex dielectric response function of a metal is negative.

For metals with high densities of conduction electrons such as silver and aluminium, the plasma frequency lies above the frequency band of visible light, in the ultraviolet region of the electromagnetic spectrum. For Lee and colleagues' conducting form of polyaniline ${ }^{2}$, however, the density of conduction electrons is much lower, and the plasma frequency is deep in the infrared, below visible-light frequencies. Polyaniline does not therefore seem to reflect visible light, as a metal does; nevertheless, its optical reflectivity in the infrared very accurately fits the model for a simple metal, and, critically, the real part of its dielectric response function remains negative down to the lowest frequencies.

In fact, the surprise is just how like an ordinarymetal polyaniline turns out tobe. Modelling its optical reflectivity assuming that it is a metal produces a realistic estimate of the number of conduction electrons (about one for every two aniline groups in the polymer), requires no adjustment to take into account the electron mass, and gives a value for d.c. conductivity that matches the directly measured value.

This finding pitches the metallic model against a different model, loosely referred to as the 'polaron model's, that over the past two decades has been the favoured explanation of polymer conductivity. This model starts from the fact that, without chemical doping, polymers such as polyacetylene and polyaniline are semiconductors (as such, they are extensively used as the active component in transistors, light-emitting diodes and photovoltaic diodes ${ }^{6}$ ). When a small amount of dopant electronic charge is added to the undoped polymer, the electronic charges on the polymer chain are localized. Thermal excitation is required to move charge from one site to the next, and so conductivity drops as temperature falls. Ironically, the localization that hinders conduction is substantially the handiwork of the agents that produced the charge: the chemical dopants are partially ordered in sites adjacent to the polymer chains (so-called 'charge transfer complexes') and their fullblown ionic charges provide random potentials that confine the electrons.

Localization is enhanced by the rearrangement near charges of covalent bonds on the polymer chain. This process produces a lower energy state for electrons to occupy, and the resultant coupling of the local structure to the electronic charge can be regarded as a particlelike entity, known as a polaron. It also causes a set of characteristic optical signatures, including vibrational modes of the polymer structure at frequencies between 15 and 60 terahertz and electronic transitions to states within the semiconductor gap, typically at frequencies in the near infrared.

Although there is plenty of spectroscopic evidence to support the polaron model for partially doped materials, polaronic features seem insignificant in Lee and colleagues' more ordered doped polyaniline ${ }^{2}$. The transition from polaronic conductor to simple metal from a material whose conductivity, at low temperatures, decreases with falling temperature to one whose conductivity increases - is surprising. The explanation must lie in the way that conduction electrons are able to screen out the local potentials that stem from both polaron geometrical relaxation and structural disorder. This process is self-perpetuating: as the potentials become screened, the conduction electrons are themselves able to become more delocalized, and thus better able to screen.

This phenomenon has parallels in some classes of inorganic materials such as 'colossal magnetoresistance' manganites that can show huge changes in conductivity as their magnetic order changes, and in which the role of polaronic interactions is similarly important ${ }^{\text {? }}$. The implications for the field of conducting polymers are bitter-sweet: although the demonstration of a polymer acting as a true metal is a real milestone, it sets a limit on the level of conductivity we can expect from such materials. Richard Friend is at the Cavendish Laboratory, JJ Thomson Avenue, Cambridge CB3OHE, UK. e-mail:rhf10@cam.ac.uk

1. Chiang, C. K et al. Phys Rev. Lett. 39, 1098-1101(1977)

2. Lee, K.et al. Nature 441, 65-68(2006).

3. Kohlman, R.S.\& Epstein A.J. in Handbook of Conducting Polymers (eds Skotheim, T.A. Elsenbaumer, R. L. \& Reynolds, L R) 85-121 (Dekker, New York, 1998).

4. Menon, R, Yoa, C. O, Moses, D.\& Heeger, A. L in Handboak of Conducting Polymers (eds Skotheim, T.A. Elsenbaumer, R. L\& Reynolds, L. R.) 27-84 (Deklker, New York, 1998).

5. Heeger, A.J.etal. Rex Mod Phys. 60,781-850 (1988)

6 Mall laras, G \& Friend, R.Phys Today 58, 53-58 (2005)

7. Littlewood, P. \& Kos, S. Nature 438, 435 (2005) 